\title{
La Biblioteca Humana Migrante: uso del espacio público y resignificación del proceso migratorio*
}

\section{The Migrant Human Library: Use of the public space and the shifting meaning of the migratory process}

\author{
Stefano Micheletti** \\ Escuela de Sociología, Centro de Estudios Urbano-Territoriales, \\ Universidad Católica del Maule, \\ Chile. \\ Sandra Vera*** \\ Escuela de Sociología, Universidad Católica del Maule, \\ Chile. \\ Javiera Cubillos Almendra**** \\ Escuela de Sociología, Universidad Católica del Maule, \\ Chile.
}

Recibido: 13 de noviembre de 2017. Aprobado: 9 de agosto de 2018.

\section{Resumen}

El presente artículo aborda la experiencia innovadora que constituye la Biblioteca Humana Migrante, realizada por el colectivo MIGRA en Talca (Región del Maule, Chile). A partir de la recopilación de información primaria y secundaria, se propone entender cuáles son los discursos que dicha experiencia promueve y cuál es el significado que ella le atribuye a la ocupación del espacio público. Los resultados dan cuenta de una acción que desde la perspectiva de los "marcos de acción colectiva" denominaremos "micromovilización", orientada a la construcción de significados integradores en el contexto del reciente flujo

* $\quad$ El artículo se construye a partir de una investigación de los/as autores/as.

** Titulado en Scienze Forestali e Ambientali, Universitá degli Studi di Padova (Italia); magíster internacional en Cooperación para el Desarrollo y Responsabilidad Social y Ambiental de la misma casa de estudios y Universidad Católica del Maule (UCM). Académico, Escuela de Sociología, UCM, investigador del CEUT. Correo electrónico: smicheletti@ucm.cl

*** Doctora en Sociología, Universidad de Barcelona. Socióloga, Universidad de Chile. Académica, Escuela de Sociología, Universidad Católica del Maule (Chile). Correo electrónico: sandraveragajardo@gmail.com

**** Doctora en Ciencia Política, Universidad Complutense de Madrid. Académica, Escuela de Sociología, Universidad Católica del Maule (Chile). Correo electrónico: jcubillos@ucm.cl 
migratorio que vive la ciudad. Por otro lado, resulta evidente el intento de esta experiencia por democratizar y repolitizar el espacio público de una ciudad intermedia de carácter agrario. Se concibe este espacio urbano como un lugar donde es posible ejercer la ciudadanía y promover - a través de actos simples de encuentro entre personas que no se conocen- la deconstrucción de estigmas y prejuicios hacia las personas inmigrantes.

Palabras clave: migración, acción colectiva, Biblioteca Humana.

\begin{abstract}
This article addresses the innovative experience represented by the Migrant Human Library, an initiative of the MIGRA collective in Talca (in the Maule Region of Chile). Using a compilation of primary and secondary sources of information, the article proposes the need to understand which discourses this experience promotes and what meaning does this attribute to the occupation of the public space. The results underline an intervention that from the perspective of "frameworks for collective action" we will refer to as "micromobilization", focused on the construction of integrated meanings within the context of the recent migratory flow experienced by the city. Furthermore, there is clearly an attempt being made through this experience to democratize and re-politicize the public space of an intermediate-sized city, one which is characterized by the role it plays in the surrounding agricultural zone. This urban space is conceived as a place where it is possible to exercise citizenship and promote the deconstruction of stigmas and prejudices towards migrants, through the simple act of bringing people together who don't know each other.
\end{abstract}

Keywords: migration, collective action, human library.

\title{
Introducción
}

El presente artículo tiene como objetivo analizar la iniciativa de la Biblioteca Humana Migrante (en adelante BHM), promovida por el Colectivo MIGRA ${ }^{1}$ en la ciudad de Talca (Chile), como estrategia de resignificación del espacio público desde la perspectiva de los marcos de acción colectiva. MIGRA se define como un colectivo intercultural que, a partir del año 2014, se ha propuesto la misión de acompañar el proceso de integración de la

1 MIGRA surge de la necesidad de ejercer una actoría social colectiva, informada y solidaria, que sirva de apoyo al proceso de integración de la comunidad migrante en la ciudad y la región. La misión que se propone el colectivo es "consolidarse como un colectivo intercultural que apoye el proceso de integración y la convivencia positiva entre la comunidad migrante y la comunidad nacional en la ciudad de Talca, Región del Maule, contribuyendo además a la promoción de los derechos humanos de migrantes, solicitantes de refugio y refugiados/as" (MIGRA, 2014, p. 1). Para mayor información, ver: https://www.colectivomigra.cl/ 
comunidad migrante - cada vez más numerosa y diversa- en Talca, Región del Maule. El sentido social y político de su reflexión/acción se ha ido plasmando en los últimos tres años en una serie de actividades, entre las que se encuentran foros y conversatorios, encuentros festivos, intervenciones artísticas y la participación en espacios de diálogo a nivel local, como la Mesa de Asuntos Migratorios del Maule. En el año 2017, el colectivo implementa una serie de BHM, con el objetivo de generar una iniciativa sociocultural comunitaria que contribuya a la integración social y al reconocimiento y respeto por la diversidad cultural en Talca, ocupando diferentes espacios públicos de la ciudad.

Con motivo de poner en valor dicha iniciativa, el artículo pretende sistematizar la única experiencia documentada de Biblioteca Humana en Chile, leerla en el marco del proceso de acción colectiva que MIGRA impulsa, y reflexionar sobre la apropiación de espacios públicos como estrategia de (re)construcción y socialización de significados, que instituye nuevos mecanismos de relación con el territorio, entendido este último como una construcción social (Di Méo, 1998; Raffestin, 1980).

El supuesto inicial entiende que las acciones colectivas desplegadas por MIGRA se insertan en un marco de acción colectiva (o frame) a nivel nacional, liderado por organizaciones como el Movimiento de Acción Migrante (MAM), ${ }^{2}$ que trata de construir significados acerca de la inmigración en Chile, reivindicando, por ejemplo, el valor de la interculturalidad, la promoción de derechos y la inmigración como oportunidad. En base a este supuesto, se analiza la experiencia de la BHM en dos ejes principales. En primera instancia, se refiere a la BHM como una "micromovilización" (Gamson, 1985), que contribuye al proceso de construcción de acción colectiva, aportando a la generación de identidad grupal, solidaridad y conciencia al interior de MIGRA. En este sentido, el artículo se pregunta sobre cuáles son los discursos que la BHM propone y cómo se vinculan estos con la realidad local. El segundo eje integra la variable territorial para atender a la especificidad de la acción que se desarrolla en el contexto de una ciudad intermedia (agraria) y utiliza el espacio público (plaza y barrio). Aquí nos preguntarnos: ¿cómo utiliza el espacio público la BHM?, ¿qué significado tiene la ocupación del espacio público en este caso?, ¿impulsa esto una resignificación del uso y la representación del espacio público?

Con el propósito de reflexionar sobre estas cuestiones, el documento se estructura en cinco apartados, además de la presente introducción. El primero aporta información de contexto sobre el flujo migratorio en la Región del Maule y Talca, junto con algunos antecedentes sobre la acción del colectivo MIGRA y la BHM, mientras el segundo se refiere al marco teórico utilizado para interpretar el objeto de estudio. La tercera sección presenta la metodología empleada para el análisis de la experiencia de la BHM y la cuarta expone los principales resultados, a saber: i) los contenidos locales que movilizan la acción de MIGRA, ii) la construcción de significados desde la BHM: discursos y resonancia, y iii) el uso del

2 Cabe destacar que MIGRA no se encuentra formalmente vinculado con el MAM. 
espacio público. Finalmente, el quinto apartado propone una serie de reflexiones a modo de conclusión.

\section{Contextualización de la experiencia: MIGRA y la migración en la Región del Maule}

Este apartado da cuenta brevemente de las transformaciones vividas en el ámbito de la migración en la Región del Maule. ${ }^{3}$ Este territorio de un millón de habitantes se encuentra ubicado al sur de la Región Metropolitana, y está conformado por cuatro provincias, siendo la ciudad de Talca el centro poblado más importante (con cerca de 260 mil habitantes) y la capital regional. Uno de los rasgos identitarios que destaca en el Maule es su carácter rural y el ser un territorio altamente productivo y exportador, especialmente en los ámbitos agropecuario y forestal (Cabello, 2015). Históricamente, la región ha recibido flujos de inmigración bastante moderados, tendencia que ha experimentado modificaciones importantes en los últimos años; es en este contexto donde nace el colectivo MIGRA.

\section{Colectivo MIGRA y el flujo migratorio en el Maule}

MIGRA es un colectivo conformado por chilenas/os y migrantes europeas/os y latinoamericanas/os, que a partir del año 2014 se ha ido constituyendo en virtud de un diagnóstico compartido entre sus integrantes acerca de los cambios que está viviendo la comunidad local en Talca y en la Región del Maule.

Coincidentemente, el comienzo de las actividades del colectivo sucede en un período en que aumenta la tramitación de visas de residencia temporaria y definitiva en la Región del Maule por parte de ciudadanos extranjeros. El incremento del flujo y la diversificación de los tipos migratorios a nivel regional ha sido abordado solo recientemente y de manera exploratoria por algunas investigaciones (Micheletti, 2016a, 2016b; Sanhueza, Salas, y Ramírez, 2016). En ellas se da cuenta de que el Maule está viviendo un proceso de transformación importante. Si bien el porcentaje de población migrante sigue siendo bajo en términos relativos, se puede apreciar un aumento constante de la cantidad de inmigrantes que arriban (y se quedan) para desarrollar su proyecto de vida (Tabla $N^{\circ}$ 1). El período 2014-2017 es de marcado crecimiento.

3 En términos administrativos, Chile se divide en 15 regiones y estas, a su vez, se subdividen en 54 provincias; estas últimas, en 346 comunas. 
TABLA N ${ }^{\circ} 1$

Población extranjera en el Maule

\begin{tabular}{|c|c|c|c|c|c|c|c|c|}
\hline Región & $\begin{array}{l}\text { Extranjeros } \\
2005\end{array}$ & $\begin{array}{l}\text { Extranjeros } \\
2014\end{array}$ & $\begin{array}{l}\text { Extranjeros } \\
2017\end{array}$ & $\begin{array}{l}\text { Aumento } \\
2014- \\
2017\end{array}$ & $\begin{array}{l}\text { Extranjero } \\
\text { vs. } \\
\text { población } \\
\text { regional } \\
2014\end{array}$ & $\begin{array}{l}\text { Extranjero } \\
\text { vs. } \\
\text { población } \\
\text { regional } \\
2017\end{array}$ & $\begin{array}{l}\text { Extranjeros/ } \\
\text { extranjeros en } \\
\text { Chile } 2014\end{array}$ & $\begin{array}{l}\text { Extranjeros/ } \\
\text { extranjeros } \\
\text { en Chile } 2017\end{array}$ \\
\hline Maule & 3.403 & 5.021 & 10.780 & $115 \%$ & $0,5 \%$ & $1,0 \%$ & $1,2 \%$ & $1,4 \%$ \\
\hline
\end{tabular}

Fuente: Elaboración propia; Instituto Nacional de Estadísticas (2018), Ministerio del Interior y Seguridad Pública (2016).

Como es posible apreciar en la Tabla $\mathrm{N}^{\circ} 2$, relativa a los datos censales publicados por el Instituto Nacional de Estadísticas (INE) en mayo de 2018, el flujo es mayoritariamente latinoamericano, y las comunidades venezolanas, haitianas y colombianas están paulatinamente reemplazando a aquellas que históricamente representaban el grueso de la migración local, como la argentina y la peruana, además de la española (Ministerio del Interior y Seguridad Pública, 2016).

TABLA No 2

Población extranjera en el Maule según país de origen

\begin{tabular}{lr} 
País & $\begin{array}{l}\text { Cantidad } \\
\text { de migrantes }\end{array}$ \\
\hline Venezuela & 1.640 \\
\hline Argentina & 1.536 \\
\hline Haití & 1.503 \\
\hline Colombia & 1.348 \\
\hline Europa & 1.156 \\
\hline Ecuador & 767 \\
\hline Perú & 665 \\
\hline
\end{tabular}

Fuente: Elaboración propia; Instituto Nacional de Estadísticas (2018).

Sin entrar en discusiones cuantitativas del fenómeno, es interesante destacar que, en una sociedad local asentada en territorio agrario, el impacto de este cambio es visible y genera consecuencias, tanto positivas como negativas, como el surgimiento de conductas discriminatorias, sobre todo hacia la población afrodescendiente.

Las/os integrantes de MIGRA -conscientes de que se trata de una temática emergente (MIGRA, 2014)- estiman que los procesos ligados a la inmigración, hasta el momento, han sido trabajados débilmente a nivel local y que se hace necesario actuar para fortalecer la 
integración entre migrantes y la comunidad chilena. A la vez, el colectivo apuesta por ocuparse de los significados sociopolíticos del proceso, entendiendo la necesidad de informarse, fomentar y difundir las discusiones que se desarrollan a partir del fenómeno de la inmigración; por ejemplo, la nueva ley de migración, el acceso a vivienda, salud y educación (MIGRA, 2014).

\section{La Biblioteca Humana Migrante}

El concepto de Biblioteca Humana (BH) nació en Dinamarca en el año 2000 gracias a la ONG Stop the Violence, cuando un grupo de jóvenes decidió hacer frente a la violencia en su país. Posteriormente, la iniciativa se ha replicado en más de 70 países para luchar contra todas las formas de discriminaciones y para promover los derechos humanos. ${ }^{4}$

La metodología de la Biblioteca Humana se ha socializado en inglés como Human Library o Living Library. Según Little y otras/os autoras/es (Little, Nemutlu, Magic y Molnár, 2011) la BH intenta desafiar los prejuicios, facilitando una conversación entre dos personas: un libro y un lector. Los libros son voluntarios que en algún momento de su vida han sido objeto de discriminación o exclusión social en ámbitos diversos, quienes traen el relato de su vivencia, dándoles permiso a los lectores para entrar en diálogo con ellos y sus perspectivas. Se desafían, así, las percepciones y los estereotipos, y por lo tanto se busca generar un cambio en las actitudes y comportamientos de la sociedad en general.

En términos prácticos, funciona igual que una biblioteca: los visitantes pueden revisar el catálogo de los títulos disponibles, eligen el libro que quieren leer y lo piden por un tiempo limitado. Después de leer, devuelven el libro a la biblioteca y, si quieren, pueden pedir prestado otro. La única diferencia es que en la Biblioteca Humana los libros son personas y la lectura consiste en una conversación (Little, Nemutlu, Magic, y Molnár, 2011). Según la asociación Human Library, la metodología ayuda a construir la comprensión de la diversidad, proporcionando un marco para sostener conversaciones reales sobre asuntos importantes.

A partir de la metodología estándar que normalmente se utiliza en la implementación de las BH (Human Library, 2016; Duffy, s/f; Little et al., 2011), MIGRA ha introducido una serie de modificaciones en dos grandes ámbitos. Por un lado, la Biblioteca Humana se ha enfocado temáticamente a la experiencia de migración (interna e internacional), sin atender a la diversidad que normalmente exige la metodología propuesta por la asociación Human Library (2016) y que define que al menos cinco de los siete "pilares de prejuicios" deben estar incluidos (etnicidad, religión, sexualidad, ocupación, estatus social, estilo de vida y salud, y discapacidades); así, los libros han centrado sus relatos en torno a vivencias

4 "Bibliotecas humanas, donde se consultan personas no libros". Disponible en: https://soybibliotecario.blogspot.cl/2016/05/bibliotecas-humanas-personas-libros.html (última fecha de consulta: 08/11/2017). 
vinculadas a su proceso de movilidad. ${ }^{5}$ Por otro lado, se ha introducido de manera intencionada la variable territorial al proceso y a su significación: el uso del espacio público ha pasado a ser un tema central para la implementación de la BH.

El colectivo ha realizado, a la fecha, cinco sesiones de la que ha denominado BHM, todas en Talca (capital regional del Maule). La primera se implementó de manera autogestionada en septiembre de 2016, en el marco de la Fiesta del Patrimonio Vivo, organizada por el Consejo Nacional de la Cultura y las Artes (CNCA). Las siguientes tres versiones de la BHM se desarrollaron entre los meses de marzo y abril de 2017, con el financiamiento del Fondo Red Cultura (fondo concursable del CNCA), dialogando en cada ocasión con una organización distinta que ha apoyado el proceso de convocatoria e implementación, a saber: las ONG locales Domodungu y Surmaule, y la Secretaría Regional Ministerial de Educación del Maule. Esta última versión se ha realizado en la Universidad Católica del Maule, en el marco del I Encuentro Regional: Debate sobre Género en la Región del Maule.

Dados los diferentes escenarios en los que se ha desarrollado la BHM, contando con el apoyo de distintas organizaciones locales, es que interesa analizar la iniciativa desde sus potencialidades de diversificar los significados en torno a la migración y el espacio público en la ciudad de Talca. En esta línea, a continuación, pasamos a exponer el marco conceptual que da origen al análisis que propone este artículo.

\section{El espacio público y sus posibilidades de resignificación desde las micromovilizaciones}

Con el propósito de exponer los principales referentes teóricos que orientan el análisis de la experiencia de la BHM desarrollada por el colectivo MIGRA en la ciudad de Talca, este apartado aborda el espacio público como un concepto dialéctico, para luego tematizar tres relatos que permiten comprender el espacio público urbano actualmente y discutir sobre las posibilidades en que las acciones colectivas, a nivel comunitario, pueden cuestionar, ampliar y/o resignificar las dinámicas que conforman y constituyen la ciudad.

\section{Espacio público como concepto dialéctico}

Para hablar de "lo público" es importante tematizar su carácter dialéctico. Como nos recuerdan Héctor Berroeta y Tomeu Vidal (2012), el espacio público vehicula a lo menos dos connotaciones. Por un lado, una dimensión política que alude a un aspecto discursivo y filosófico (esfera pública); y, por otro, una dimensión urbana, que hace referencia a la ciudad y su conformación morfológica (espacio urbano). Dichas connotaciones -fuertemente

5 También ha generado una serie de pequeñas innovaciones metodológicas que, al no ser centrales para los fines de este artículo, no serán detalladas. 
interrelacionadas al punto de que no puede entenderse la una sin la otra- dan cuenta del modo en que la sociabilidad (la convivencia común) moldea la organización de la ciudad y, al mismo tiempo, cómo el ordenamiento de la ciudad (de)construye (controla, reproduce y/o transforma) el discurso, el encuentro y la interacción social.

La dimensión política nos recuerda que, a la base del espacio público urbano, está la asociación y el diálogo entre sujetos que se asumen libres e iguales (ciudadanos), para el establecimiento de acuerdos que les permitan una convivencia pacífica. De esta manera, la ciudad puede ser comprendida como un espacio donde se satisfacen ciertas necesidades y se ejercen determinados derechos. Entre tales necesidades y derechos, se destaca la participación - entendida como la capacidad y la posibilidad de intervenir (por ejemplo: criticar, comunicar, proponer, decidir, etc.) lo público (lo compartido)-, la que permite el acceso y la optimización de ciertos recursos y beneficios asociados a la satisfacción de [otras] necesidades" (Alguacil, 2008, p. 202); y posibilita el ejercicio de ciudadanía a través de la apropiación de la urbe y la interacción con otras personas y grupos, con otras visiones e interpretaciones de la ciudad y lo público.

Desde una perspectiva filosófica, Hannah Arendt destaca el carácter de la esfera pública en tanto mundo que es común a todos nosotros "y diferenciado de nuestro lugar poseído privadamente en él”" (2009, p. 61). Es decir, la esfera pública "nos junta y no obstante impide que caigamos uno sobre otro" (Arendt, 2009, p. 62).

En la dimensión urbana de lo público, la ciudad puede ser entendida como un territorio donde se dramatiza lo político y tiene lugar la performance ciudadana; un escenario de exposición y visibilidad generalizada donde se expresa la heterogeneidad, se coincide con otras/os y se (re)produce un tipo específico de sociabilidad (Arendt, 2009; Borja y Muxi, 2003; Delgado y Malet, 2011). La ciudad -como expresión de lo público- sería un ámbito que posibilita lo humano, en tanto individualidad y colectividad (Arendt, 2009; Volante, 2011). El espacio público, metaforizado en la ciudad, puede ser comprendido como un territorio de coexistencia de la heterogeneidad social, donde "se conforma y se confirma la posibilidad de estar juntos" (Delgado y Malet, 2011, p. 58), en base a acuerdos (que se asumen) colectivos.

Comprendiendo esta doble acepción, es posible afirmar que la interacción social construye ciudad y, a la vez, la ciudad modela sociabilidad. En este sentido, ambos espacios (político y urbano) pueden ser vistos como un diálogo societal, no exento de conflictos y tensiones, en constante construcción y deconstrucción del que -por presencia o ausencia, por acción e inacción- todas/os somos parte. Es en "lo público" donde renovamos cotidianamente los acuerdos colectivos, una renovación que implica tanto la reproducción como la refutación de las normas de convivencia compartidas. 
Tres narraciones para comprender el espacio público urbano en la actualidad

De la mano de esta doble connotación de espacio público, Héctor Berroeta y Tomeu Vidal (2012) retoman tres corrientes teóricas para aproximarse al entendimiento del espacio público en la ciudad actual, a saber: i) espacio público como pérdida, ii) espacio de construcción de civilidad, y iii) espacio de control y disputa. A continuación se abordan algunos elementos de estas tres propuestas que posibilitarán acercarnos a la experiencia de la BHM.

\section{El espacio público perdido}

La concepción que plantea la pérdida del espacio público denuncia la disolución de la ciudad a partir del fenómeno de privatización urbana caracterizado por la "homogenización de la ciudad, [la] hipervigilancia y [el] control espacial" (Berroeta y Vidal, 2012, p. 11). Esta representación asume, por un lado, que el espacio público en algún momento anterior fue democrático, es decir, construido sobre los valores de igualdad, libertad y respeto de la diferencia -supuesto que será cuestionado por otras perspectivas teóricas (ver más abajo en subtítulo "Espacio público como lugar de control y disputa")-; y, por otro lado, entiende que este espacio público democrático - con su respectivo correlato urbano - ha sido sustituido por la segregación urbana y la sofisticación en los mecanismos de seguridad, que reproducen dinámicas sociales desiguales. Estos fenómenos serían observables en la transformación de la ciudad, en los patrones, hábitos y rutinas de circulación y en el uso de espacios comunes, evidenciándose un repliegue desde los espacios públicos a los espacios privados y pseudopúblicos, como centros comerciales y condominios cerrados (Berroeta y Vidal, 2012). Dicho repliegue estaría dado por la construcción social de la inseguridad, que representa el espacio público urbano como amenazante y hace perder de vista la solidaridad, el interés y el respeto hacia las/os otras/os (Segovia y Dascal, 2000).

\section{Espacio público como construcción de civilidad}

La perspectiva que representa el espacio público como espacio de construcción de civilidad, sin descartar necesariamente la visión anterior, reconoce que el "espacio público es el principal espacio del urbanismo, de la cultura urbana y la ciudadanía, cuya calidad, multiplicación y accesibilidad dependen en buena medida del progreso de la ciudad" (Berroeta y Vidal, 2012, p. 12). Visto así, se posiciona la necesidad de proyectar un espacio público mejor.

Al interior de este enfoque pueden identificarse dos énfasis distintos (Berroeta y Vidal, 2012): la tradición estadounidense y la europea. En el marco del artículo interesa rescatar la llamada tradición europea, que plantea que la ciudad contemporánea se enfrenta a nuevos desafíos, siendo crucial pensarla y construirla activamente. Esta perspectiva repolitiza la ciudad -en base al modelo citadino europeo- al construirla como condición básica y espacio 
propicio donde ejercer la ciudadanía. La ciudad sería, entonces, un derecho humano fundamental y, por tal, es necesaria su recuperación y su dotación de equipamiento. Entre otros aspectos, por ejemplo, apela a ofrecer centralidad a la llamada periferia urbana.

\section{Espacio público como lugar de control y disputa}

Finalmente, la corriente teórica que representa el espacio público como espacio de control y disputa se plantea críticamente ante la concepción romántica de un espacio público igualitario (ver más arriba en subtítulo "El espacio público perdido"), afirmando que lo público dista de ser un territorio armónico, al propiciarse dinámicas de exclusión fundadas en lógicas de control y disputa entre la estructura normativa de la ciudad hegemónica (el orden político dado por la administración y la proyección centralizada de la ciudad) y las prácticas de quienes componen la ciudad (los tránsitos y coaliciones temporales entre transeúntes) (Berroeta y Vidal, 2012; Delgado, 2001). El espacio público, como otros constructos sociales, no sería neutral, sino que estaría mediado por la ideología de los grupos sociales dominantes. La ilusión (o ideología) ciudadanista, como la llaman Manuel Delgado y Daniel Malet (2011), propondría una especie de democraticismo radical en la perspectiva de realizar empíricamente el proyecto moderno (en su dimensión política), a la vez que ocultaría -bajo el velo de la universalidad- una jerarquía de valores y significados que representan los intereses de las clases dominantes. La ciudad sería, entonces, la concreción física en la que se dramatiza la ilusión ciudadanista (Delgado y Malet, 2011).

Al vincular estos argumentos con ciertos postulados de la teoría feminista, es posible afirmar que el espacio público promovido por las democracias liberales se sustenta en la idea de un sujeto particular (hombre, adulto, heterosexual, burgués, occidental-blanco) asumido como universal, lo que oculta relaciones de poder y dinámicas de exclusión específicas (Alexander y Mohanty, 2004; Benhabib, 2006; Brah, 1996; Fraser, 1990a, 1990b, 1997, 2006; Okin, 1989; Young, 1990, 1996). En este escenario, no todas las personas tendrían igual libertad (o derecho) de apropiarse del espacio público, tanto en su concepción política como urbana. Ciertos discursos, representaciones y prácticas suelen ser marginadas o construidas como periféricas en la ciudad. No todos quienes la habitan tienen iguales posibilidades de construir la convivencia común. Las posibilidades de interacción y diálogo en la ciudad se verían limitadas por las estructuras de desigualdad que articulan lo público, debilitando la participación de ciertos sujetos en las dinámicas citadinas. No todos estaríamos llamados a crear ciudad ni seríamos reconocidos como interlocutores válidos (sujetos libres e iguales) para construir el consenso colectivo. En este sentido, la ciudad está lejos de ofrecer iguales posibilidades de visibilidad e interacción.

Entre los sujetos que no estarían en igualdad de condición para habitar o circular por ciertos lugares de la ciudad, podrían encontrarse las mujeres y las personas migrantes. En el caso de las mujeres, uno de los mecanismos de control de su movilidad sería el acoso callejero, materializado en gestos obscenos, ofensas verbales, miradas lascivas, acoso físico, 
exhibicionismo, entre otros (Gaytan Sánchez, 2009; Vallejo y Rivarola, 2013). Por su parte, la marginación de las personas migrantes de las dinámicas de la ciudad se vincula con la tendencia a instalarse en las periferias de las ciudades de acogida, lo que conlleva una merma en su calidad de vida, sobre todo en ciudades chilenas caracterizadas por una marcada segregación socio-espacial (Aymerich, 2013; Sabatini, Cáceres, y Cerda, 2001; Sabatini y Soler, 1995). Los grupos migrados que residen en la periferia urbana suelen ser criminalizados, tanto por la policía como por la ciudadanía, por lo que su presencia en lugares públicos suele ser estigmatizada, al ser representada como incómoda y como factor de inseguridad (Borja y Muxi, 2003).

No obstante, desde lugares de exclusión urbana o desde posicionamientos críticos a la composición de la ciudad, emergen nuevas apuestas para pensar tanto lo político como lo urbano. Los transeúntes serían, entonces, ejecutores de actos creativos que pueden resignificar la ciudad, al apropiarse de los espacios públicos y reivindicar su derecho a la ciudad (Lefebvre, 1978). Quienes habitan la ciudad no solo viven los efectos de su planificación, sino que pueden optar por una performance política antagónica comprendiendo el espacio público como plataforma de disenso político-, con intención de (re)crear vínculos de sociabilidad más igualitarios (Berroeta y Vidal, 2012). En este escenario, la representación de "lo público" puede refutarse, ampliarse e integrar nuevos significados, contribuyendo a renovar -o rechazar- el consenso social y las normas que articulan la convivencia común.

Espacio público como debate: la ampliación o resignificación de "lo público" desde espacios microsociales

En nombre de la convivencia pacífica, muchas veces la diferencia, la disidencia y el conflicto son negados, asumiéndose que solo en la homogenización de pareceres es posible la gobernabilidad. En este entendido, y parafraseando a Manuel Delgado y Daniel Malet (2011), podríamos afirmar que el espacio público vehicula también un mecanismo ideológico donde quedan cancelados los antagonismos sociales. En este contexto de anulación del conflicto social y de asimilación de la divergencia, diversos grupos sociales conscientes de su diferencia - una diferencia excluida de las dinámicas hegemónicas de la ciudad y lo políticoplantean procesos de reafirmación identitaria, demandan reconocimiento y generan propuestas para pensar lo público y la interacción social desde la heterogeneidad social (Fraser, 1990a, 1990b, 2006; Hill Collins, 2009; Young, 1990, 2000). Dicha demanda por reconocimiento social implica una renovación del consenso respecto de los derechos y deberes que son (o no) concedidos y por la posibilidad/capacidad de habitar el espacio urbano común, como sujetos políticos y transeúntes activos.

Desde esta demanda (implícita o explícita), y desde dicha reafirmación identitaria, podemos aseverar que los diferentes grupos sociales tienen la capacidad de irrumpir en el espacio público, renovando los discursos circulantes y la representación misma de lo público. En este 
entendido, los marcos de acción colectiva nos permiten un acercamiento interesante para comprender la intervención de la BHM propuesta por el Colectivo MIGRA en lugares públicos de la ciudad de Talca.

Los marcos de acción colectiva, asociados a la teoría del frame analysis que surge en la década de los ochenta, plantean la revalorización de la dimensión simbólica de la acción colectiva en los procesos de construcción social (Acevedo, 2013; McAdam, McCarthy y Zald, 1999). Un frame (marco interpretativo o marco de acción colectiva) puede ser entendido como un conjunto de creencias y significados que orientan y legitiman la acción de los movimientos sociales (Benford y Snow, 1994). Estas estructuras de sentido sirven de encuadre a la acción colectiva, representando una situación que se asume problemática (diagnóstico), sus posibles causas y agentes responsables (antagonistas), desde donde se definen posibles estrategias de acción que permitan resolver el problema representado (pronóstico), identificando los agentes (protagonistas) de la transformación social (Benford y Snow, 1994; McAdam et al., 1999; Zald, 1999).

Un marco de acción colectiva (frame) guarda una interpretación particular de la realidad social (Benford y Snow, 1994; Chihu Amparán, 2012; Gamson, 1992; McAdam et al., 1999; Snow y Benford, 2006; Zald, 1999). Un frame surge cuando los integrantes de un movimiento social llegan a compartir la definición de un problema, acuerdan que dicha situación amerita ser transformada y se adjudican la agencia colectiva para subvertir los marcos tradicionales de acción política. Este ejercicio discursivo trae al debate público sentidos y significados que permiten ampliar las concepciones de justicia, al identificar un problema social representado como injusto. El levantamiento de un frame es un esfuerzo estratégico de un grupo de actores para legitimar las acciones de un movimiento social y motivar, a una determinada audiencia, a la acción colectiva. Se busca generar un compromiso con la acción transformadora, convenciendo a otros (audiencia) -representados como potenciales protagonistas del cambio social- para la consecución de las soluciones (Chihu Amparán, 2006). De este modo, los marcos de acción colectiva sirven a los movimientos sociales para asignar significado e interpretar los acontecimientos sociales, con la finalidad de movilizar a simpatizantes y desmovilizar a sus antagonistas. La resonancia del proceso de enmarcamiento de un movimiento se entiende como la posibilidad de que las acciones desplegadas logren implicar y movilizar a una población (Snow y Benford, 2006).

Si asumimos que los esfuerzos de movilización se componen de una serie más o menos sistemática de interacciones, para el análisis presentado parece especialmente interesante el concepto de micromovilización (Chihu Amparán y Gallegos, 2004), que puede ser entendida como una serie de "encuentros", la unidad de análisis más pequeña dentro del orden de la interacción. Específicamente se trata de: una reunión enfocada, es decir, dentro de un "encuentro" la interacción posee un único foco de atención, un objetivo único y claro para los participantes; los actores poseen una elevada conciencia de la relevancia mutua que poseen los actos de cada uno de ellos; las actividades realizadas por los participantes se 
"enfocan", es decir, ponen especial atención a sus actividades con la finalidad de que transmitan un solo y único mensaje que ha de ser captado de manera unívoca por los otros actores; y las actividades que se producen en un "encuentro" tienen un comienzo y un final sumamente marcados por algún tipo de ceremonia o ritual.

\section{Metodología}

El análisis que exponemos se enmarca en un paradigma constructivista en la medida en que buscó reconstruir el proceso de diseño, construcción y significación de la experiencia de la BHM, desde la perspectiva de los actores involucrados. Para esto se desarrolló un estudio cualitativo, basado principalmente en la aplicación de entrevistas semiestructuradas a quienes coordinaron el proyecto y a quienes participaron del proceso en calidad de libro humano (no se trabajó con quienes participaron en calidad de lectores).

En primer lugar, se realizó un seguimiento permanente a la iniciativa -desde la etapa de diseño hasta la implementación y evaluación-, participando en todas las actividades de la $\mathrm{BHM}^{6}{ }^{6}$ incluyendo una evaluación intermedia que fue grabada y utilizada como material de análisis. En segundo lugar, se aplicaron dos entrevistas semiestructuradas a los coordinadores de la iniciativa (una mujer italiana y un hombre francés) y una entrevista grupal a cuatro migrantes de diferentes nacionalidades (Chile, ${ }^{7}$ España, Italia, Uruguay), quienes participaron de la experiencia como libros humanos y también cumplían con el criterio de participar en el colectivo MIGRA, para poder rescatar su visión más integral acerca del proceso. De forma complementaria, se realizó una revisión de material escrito y audiovisual correspondiente a noticias y videos sobre MIGRA y el proceso de la BHM, que permitió integrar al análisis el relato de migrantes latinoamericanos que actuaron como libros, pero que no pudieron participar de las entrevistas presenciales.

Toda la información recolectada ${ }^{8}$ fue sistematizada en matrices de integración (codificación temática). Posteriormente se procedió al análisis, que utilizó parcialmente la metodología frame analysis. Las unidades de análisis son textos producidos por los actores involucrados en la BHM, buscando indagar en el discurso de los protagonistas como un proceso de negociación y construcción de la realidad social. El frame analysis se usa en la (re)construcción y negociación de la realidad por parte de actores sociales y políticos, donde

6 Las actividades asociadas a la BHM son las siguientes: el diseño de la iniciativa, la postulación al Fondo Red Cultura del Consejo Nacional de la Cultura y las Artes, la planificación de las actividades, la convocatoria, la capacitación de los participantes, la implementación de las bibliotecas y la evaluación del proceso.

7 No solamente personas extranjeras participaron de la experiencia, sino quienes también han experimentado el proceso de migración en su vida (por ejemplo, migrante interno o desde/hacia otros países).

8 Cabe destacar que el proceso de recopilación de la información contó con el consentimiento informado por parte de los involucrados en la investigación. 
se busca identificar los marcos dominantes y/o en conflicto en el discurso de los actores que dan sentido a diferentes situaciones, asignar significado e interpretar acontecimientos sociales, atribuir culpas o causalidades y proponer líneas de actuación (Bustelo y Lombardo, 2007).

\section{Análisis de la experiencia de la BHM}

El propósito de este apartado es exponer los principales elementos detectados en el contexto del análisis de la información recolectada sobre la BHM y el colectivo MIGRA. Para su exposición, el apartado se ha dividido en tres secciones -que abordan las percepciones de MIGRA que movilizan su acción, la construcción de significados desde la implementación de la BHM y el uso del espacio público en miras a su resignificación-, a modo de relevar los aspectos que articulan la acción de MIGRA.

Las percepciones del colectivo MIGRA: los contenidos locales que movilizan la acción

El aumento en el flujo migratorio y la diversificación de los perfiles de los extranjeros que han llegado a la ciudad de Talca, especialmente a partir del año 2014 (Micheletti, 2016a, 2016b), han contribuido a dibujar un paisaje sociodemográfico local con elementos nuevos. Las percepciones de los integrantes de MIGRA sobre este proceso constituyen el diagnóstico inicial que motiva su movilización como colectivo. De hecho, en el plan estratégico del colectivo se declara que:

Se hace necesario actuar para fortalecer la integración entre migrantes y comunidad chilena, creando espacios de encuentro para compartir lo cotidiano, las tradiciones, las historias, etc., así como orientar a las personas que lo necesitan en el proceso de ingreso, estadía, residencia y egreso, a partir de nuestras experiencias. (MIGRA, 2014)

Si bien se trata de un diagnóstico general, para el colectivo representa una declaración inicial que estructura y orienta la acción, y que le permite reconocerse como un actor relevante para revertir la problemática y plantear estrategias (el encuentro, el acompañamiento) para su resolución. Para analizar con mayor profundidad los alcances de este diagnóstico, y con el fin de identificar con más claridad cuál es el "problema" que moviliza al colectivo, se pueden considerar las percepciones de las/os entrevistadas/os sobre los cambios en el flujo migratorio y la reacción de la comunidad de acogida.

En primer lugar, las personas entrevistadas comparten la sensación de que existe un aumento importante en el arribo de extranjeros, y que esta dinámica está ligada a un proceso de orden global que tiene expresiones locales. 
Existieron importantes migraciones, pero no era así como hoy día, yo creo que estamos en un cambio de paradigma tremendo de que es como se vive la vida en este planeta, estamos tomando conciencia que es un globo flotando y que se puede, se puede migrar acá, se perdió un poco la distancia. (Alicia, Uruguay, 8 de junio, 2017)

La "pérdida de las distancias" - entre otros factores- hace que territorios antes no contemplados entren en el mapa de las rutas migratorias, donde "Talca no es ausente de este nuevo movimiento" (Cedric, Francia, 26 de mayo, 2017). Es entonces importante reconocer las características propias de este fenómeno.

En los últimos tres años, yo diría, la migración es una migración siempre más negra y no es solo negra, sino que es una migración que tiene otro idioma (...). Lo novedoso que está pasando en el Maule y seguramente en Chile, es que por primera vez hay que acostumbrarse a otro tipo de idioma y no hay que pensar que quien llega aquí habla necesariamente castellano. (Emilia, Italia, 26 de mayo, 2017)

Según las/os integrantes de MIGRA, las principales motivaciones para desplazarse desde sus países de origen dicen relación con la búsqueda de una mejor calidad de vida: "vinieron aquí por razones económicas, por ejemplo, y todo lo que hacen aquí es trabajar para mandar plata para allá, para mandar cariño del otro lado, para viajar lo más pronto posible de vuelta o hacer idas y vueltas" (Cedric, Francia, 26 de mayo, 2017).

Sin embargo, la estructura de oportunidades de las/os inmigrantes -que es en general reducida- se vuelve mínima en algunos casos específicos. Así, la inserción en la sociedad local se concreta de manera segmentada en términos laborales y espaciales.

Lo que pasa es como la sociedad es segregada y la ciudad es segregada, los haitianos, digo los inmigrantes pobres o de lugares que en Chile se ven como más marginales están todos concentrándose en la zona norte, entonces, claro, en este caso se nota mucho que son inmigrantes "porque son morenos, porque hablan otro idioma". (Loreto, Chile, 8 de junio, 2017)

El componente de la "negritud" (Césaire, 1994; Fanon, 2009) hace más visible a las/os inmigrantes, y esa "marca" termina representando un estigma construido por la comunidad nativa. Para Loreto (Chile, 8 de junio, 2017), "en general Chile es una sociedad clasista y que discrimina (...), no es lo mismo venir de Europa que venir de El Salvador, de Guatemala o de Haití; se establecen como en otra categoría”. De acuerdo a Cedric (Francia, 26 de mayo, 2017), se puede "percibir en el ambiente que es una tierra donde no veías nunca una persona negra y ahora es cotidiano, y se siente la molestia o el cambio que la gente de aquí puede tener con esta aparición". 
La discriminación -racismo en algunos casos- fortalece la construcción de una sociedad y de un territorio segregado. En esta línea, Giorgio (Italia, 8 de junio, 2017) agrega: "es muy fuerte, tenemos gente, haitianos y, en estos momentos, algunos encuentran trabajo, pero la mayoría no se sabe dónde después van. Es un tema muy fuerte porque están llegando a barrios populares $(. .$.$) es para preocuparse".$

Las percepciones expresadas por los/as entrevistados/as dan cuenta de cómo la ciudad vivida genera indicios claros acerca de una transformación que se está dando; junto a la sensación de que hay más inmigrantes y que el componente negro es cada vez más evidente, hay una preocupación explícita acerca de las posibilidades reales de integración de la comunidad migrante, especialmente de aquellos que son estigmatizados por su país de origen y su color de piel.

Esta interpretación compartida constituye el núcleo de significados centrales del colectivo, asociado al problema social o injusticia detectado/a, que es el primer paso para la identificación de posibles estrategias de acción colectiva para darle solución. En nuestro caso de estudio $-\mathrm{y}$ ya declarada la voluntad de agencia colectiva de MIGRA-, el proceso desemboca en el levantamiento e implementación (entre otras acciones) de la BHM.

\section{La construcción de significados desde la BHM: discursos y resonancias}

La interpretación que realizan las personas entrevistadas es clave para entender el significado atribuido a la BHM, y también los discursos (más o menos explícitos) que emergen en el ejercicio de diálogo con el "otro". Para Cedric (Francia, 26 de mayo, 2017), "esta herramienta simple y sencilla de sentarse a conversar, es una acción de paz que yo creo genera muchas cosas para 'los libros'; sentir que la gente está dispuesta a escucharte". De acuerdo a Giorgio (Italia, 8 de junio, 2017), "la Biblioteca es afrontar un tema emocional, de relación con la población chilena”. En esta línea, Loreto (Chile, 8 de junio, 2017) expresa que la BHM es "un espacio de contención mutua". Y en palabras de Fermín (España, 20 de mayo, 2017), "en este espacio de diálogo que se da, con personas que no conoces, estableces realmente un nexo y una comunicación efectiva".

La propuesta de la BHM, lejos de la construcción de discursos generalizadores y generalizables, apunta a experiencias personales (en la dinámica de conversación con los "libros"). Aquello podría ser entendido, desde Snow y Benford (2006), como un factor clave para la posibilidad de resonancia cultural. Específicamente en las restricciones fenomenológicas que afectan las posibilidades de movilización de los marcos, toda vez que requieren una "afinidad con la experiencia", es decir, que el enmarcado no resulte demasiado distante o abstracto "respecto a las experiencias cotidianas de los participantes potenciales" (Snow y Benford, 2006, p. 104). Como expresa María (Venezuela, 20 de mayo, 2017): 
Merece que más gente conozca lo difícil que es a veces para nosotros los migrantes o nuestra experiencia, la verdad es que la gente debe conocer lo que vivimos nosotros también para que ellos el día de mañana si le toca a alguno salir, que vea que sí le cuesta no solo a él, sino que hay más personas como él.

Resalta en una primera lectura la dimensión íntima de la experiencia (elemento muy llamativo de análisis, pues esta "intimidad" se construye en un espacio público). Sin embargo, aparece posteriormente -aunque de forma no siempre explícita y consciente- el componente político de la acción. Por un lado, a través de la afirmación del "yo" como acto político fundamental y, por otro, visualizando los efectos de integración que se pretenden generar. Es la afirmación de un "yo" que deviene en un "nosotros" -expresada en una interacción específica-, la que instala un hecho político de mutuo reconocimiento que se expresa y tiene cabida en el espacio público:

Lo que queremos hacer es encontrarnos, porque en un acto tan simple como sentarse a conversar, (...) es lo que se necesita para que el migrante y el nacional, o el migrante e inmigrante, o el migrante con los que han inmigrado, con el viejo, con la joven, se acerquen desde un relato humano. (Emilia, Italia, 26 de mayo, 2017)

Es evidente que se apunta al encuentro como estrategia de resolución (o reducción) de la problemática identificada; esto, sin determinar a priori el efecto que se quiere generar a través de la conversación entre el "libro" y el "lector", sino más bien dejando que la conversación misma moldee los sentidos de este encuentro, y deje como enseñanza final que el conocerse contribuye a disminuir los miedos, las agresividades y los prejuicios asociados a los inmigrantes. Alicia (Uruguay, 8 de junio, 2017) lo expresa de la siguiente manera:

En general, las personas discriminan lo que no conocen o lo que temen, o lo que les resulta extraño, en el momento de que conoces deja de parecer extraño, dejas de temer, la posibilidad de discriminar, y ver al otro como un verdadero otro y no como una cosa o un objeto... los motivos para discriminar, aunque no sean verdaderos motivos, ya no deberían estar presentes. Creo que ese es el aporte.

En relación con los sujetos y actores interpelados a través de este encuentro, el foco principal está puesto en la comunidad chilena en cuanto destinataria del mensaje/discurso propuesto. Al respecto, Enrique (España, 8 de junio, 2017) comenta: "La idea era de llevar a los chilenos esta temática, hacer conocer otras historias y a la Plaza de Armas fue fuerte, porque fue mucha persona que quería escuchar".

En el desarrollo de las bibliotecas, los libros humanos encuentran receptividad especialmente con el grupo de las/os niñas/os y las/os jóvenes. Como expresa Giorgio (Italia, 8 de junio, 2017), el "ser libro fue de verdad, más con los jóvenes, un encuentro muy bello, de mucha 
curiosidad del otro lado". Loreto (Chile, 8 de junio, 2017), en la misma línea, expresa: "me llamaba mucho la atención que fueran los niños que en cierta forma arrastraban a los adultos".

\section{La ocupación del espacio público y sus posibilidades de resignificación}

La BHM interviene el espacio público de una manera festiva, en un contexto local donde prima la lógica "puertas adentro", no solamente vinculada al espacio privado/doméstico, sino también a una forma de sociabilidad que se construye alrededor de las organizaciones comunitarias (como Juntas de Vecinos, clubes deportivos y centros culturales), cuyas actividades se centran principalmente en el bienestar de sus afiliados. Al respecto, Emilia (Italia, 26 de mayo, 2017) manifiesta: "Esta Biblioteca Humana Migrante se hace en espacio público (...), porque entendemos la importancia de ocupar un espacio que en [las] dos ocasiones fue bastante central y que fuera "una bomba".

Si bien no se reconoce como un objetivo explícito de la iniciativa, la BHM permite la recuperación de espacios públicos a través de una actividad de corte sociopolítico gestionada por una organización de la sociedad civil. Como comenta Alicia (Uruguay, 8 de junio, 2017):

Lo que falta es que las personas se apropien del espacio público, de la plaza que tienen en su barrio. Ahí tú haces vida, no solamente te apropias del espacio público, haces más vida vecinal (...). Se van creando redes vecinales. A ese espacio público yo le encuentro más sentido.

El encuentro, así comprendido, se refuerza como lugar fundamental para construir la "esfera de los asuntos humanos" (Arendt, 2009, p. 207). El mundo y la esfera pública existen ante las personas debido a la existencia de interacción, es decir, a que "actúan y hablan unos para otros" (Arendt, 2009, p. 207). En ese sentido, la esfera pública -entendida como "lo común"se relaciona con que "todo lo que aparece en público puede verlo y oírlo todo el mundo, y tiene la más amplia publicidad posible (...) algo que ven y oyen otros al igual que nosotros constituye la realidad" (Arendt, 2009, p. 59).

La interacción en y la intervención de "lo público" supone, en este caso, una puesta en escena que quienes organizan la BHM reconocen como elemento central, y que han trabajado desde el comienzo, incluido el proceso de formación de los libros:

La persona que forma los libros (...) es una persona que hace teatro de calle, entonces 'al toque' nos dio herramientas para que la gente se entere más fácilmente, para ir a buscar a la gente, para invitar, para dar confianza, para habitar el espacio. (Cedric, Francia, 26 de mayo, 2017)

El escenario está preparado escrupulosamente, y se disponen sillones, cojines y artículos decorativos para hacer más acogedor el espacio; los detalles cobran relevancia: 
Nos preparamos, nos 'amononamos', nos ponemos la camiseta, ponemos letrero, marcamos un territorio y decimos 'este que es el espacio público, sirve también para hacer cosas', y es como el espectáculo, entonces se hace. La gente también entra en ese juego. (Loreto, Chile, 8 de junio, 2017)

Aquí se destacan dos aspectos. Por un lado, un sincretismo entre "lo íntimo" y "lo público" - creando una especie de tercer espacio de sociabilidad- y, por otro, una performance colectiva que busca alterar el statu quo, las rutinas de la ciudad y la vida pública. Un momento liminal (Van Gennep, 1960; Turner, 1997) en el cual se hace posible la renovación simbólica de la comunidad y la sociabilidad misma. Las sombrillas y los cojines reparan y acogen, los relatos y las conversaciones fluyen, y de manera espontánea se constituyen como actos de resistencia a la discriminación, la segregación y la exclusión, pero también a la pérdida de lo “común”. Así lo expresa Loreto (Chile, 8 de junio, 2017): "Que la gente está volviendo a ocupar sus espacios y hay que echarle mano a eso, aprovecharla y hay una bella relación de volver a tomarlo, a hacerlo propio, sentirlo más parte de la vida”.

Es una resistencia a lo que Arendt (2009) define como el "fin del mundo común", que no se produce solo por tiranías y dictaduras, sino también por el encierro en la propia experiencia singular y subjetiva, cuando:

los hombres se han convertido en completamente privados, es decir, han sido desposeídos de ver y oír a los demás, de ser vistos y oídos por ellos. Todos están encerrados en la subjetividad de su propia experiencia singular, que no deja de ser singular si la propia experiencia se multiplica innumerables veces. (Arendt, 2009, p. 67)

\section{Reflexiones finales}

Para abrir este apartado de reflexiones queremos destacar, en primer lugar, que la BHM se interpreta como una micromovilización, y en ningún caso se entiende al colectivo MIGRA como un movimiento social con capacidad de construir un marco de acción colectiva; más bien, representa una contribución a un frame de nivel nacional que construyen organizaciones más grandes. Luego, nos parece importante señalar que la experiencia tiene un conjunto de características muy vinculadas a los movimientos sociales actuales: i) no se constituye en torno a las demandas de una clase social, sino de una categoría temática (que podríamos identificar como "migración"); ii) en relación a los contenidos introduce discursos que no están asociados a los paradigmas "tradicionales", como las condiciones de trabajo, la distribución económica, etc.; y iii) en cuanto a los modos de actuar se caracteriza por utilizar formas nuevas, bastante alejadas de las marchas o protestas: una intervención ciudadana "intimista", basada en el encuentro y el diálogo. Sobre este último punto cabe resaltar el uso 
del "encuentro" como instrumento central: un encuentro directo entre personas que no se conocen, simple en su forma y sin mediación de terceros.

Si nos remitimos a las preguntas formuladas al comienzo del artículo, el análisis de las entrevistas y el material recopilado permiten identificar la existencia de un conjunto de discursos que MIGRA trata de vehicular a través de la BHM en el espacio público. Estos discursos, generados a partir de la preocupación por las expresiones de discriminación que acompañan el proceso de inserción en la comunidad local de los inmigrantes, se construyen a través del encuentro intencionado entre personas que no se conocen y que transitan en la ciudad. La interculturalidad como valor, la migración como oportunidad y la comunión de las historias personales son mensajes que aparecen de forma permanente en los relatos de las personas entrevistadas.

La experiencia se puede interpretar, entonces, desde la perspectiva de un espacio público que vehicula mecanismos ideológicos donde se trabajan los antagonismos sociales. En este caso, y como hemos planteado en el marco teórico, la BHM representa la expresión de un grupo social consciente de su diferencia - una diferencia excluida de las dinámicas hegemónicas de la ciudad y lo político-, que plantea procesos de reafirmación identitaria y demanda reconocimiento, proponiendo prácticas y discursos para renovar las dinámicas de sociabilidad.

Valgan aquí dos reflexiones. En primer lugar, es interesante observar cómo el ejercicio de la BHM -si bien es una acción que no se instala permanentemente- representa un intento claro por democratizar el espacio público. No solamente todas/os tienen derecho de estar y contar su historia de vida, sino también todas/os tienen el derecho de escuchar y dialogar.

En segundo lugar, el espacio público se repolitiza al entender la ciudad como el lugar donde ejercer la ciudadanía, una ciudadanía que se construye a través de una micromovilización asociada a la capacidad de agencia de un colectivo. Queda en evidencia aquí la importancia de la acción colectiva como instrumento con un fuerte contenido político (aunque no constituido en un objetivo explícito de la BHM), en contraposición con la pasividad, pero también con los espacios de participación que el Estado "ofrece", y que muchas veces son inocuos en términos de la posibilidad de generar e instalar discursos propios.

Acciones como estas pueden entonces entenderse como una de las (muchas) voces que consideran que el espacio público es interacción; diferenciándose de quienes lo entienden como un lugar en la esfera política en que sería posible renunciar a esta, lo que -desde una lectura arendtiana- "quita a la vida política su sentido existencial y lo carga de una connotación meramente instrumental” (Volante, 2011, p. 102).

Por último, queda como desafío investigativo situarse en la posición de quienes cumplen el rol de "lector/a" en la intervención de la BHM. Al respecto, se puede advertir que la dinámica 
del "encuentro" antes expuesta no significa automáticamente que las personas que participan como lectores en una intervención de este tipo modifiquen sus percepciones o representaciones respecto de quienes migran y/o hacia ciertas experiencias migratorias. En esta línea, se abre la pregunta sobre la forma en que serían recibidos los relatos de migración más controversiales - pero no por eso inexistentes-, que dieran cuenta de rutas migratorias menos legitimadas y con potencial más conflictivo para la escucha. Probablemente, aquel escenario excede las posibilidades de la BHM tal como ha funcionado hasta el momento y/o desafiaría los límites en la profundidad del encuentro, abriendo la oportunidad de poner en la conversación asuntos más incómodos, pero también más nutritivos y complejos para la construcción de significados en torno a la migración.

\section{Referencias}

Acevedo, M. (2013). Principales críticas conceptuales al Frame Analysis: del Frame al Framing. Pilquen - Sección Ciencias Sociales, 16(2), 1-14.

Alexander, M. J. y Mohanty, C. T. (2004). Genealogías, legados, movimientos. En B. Hooks, Otras inapropiables: feminismo desde las fronteras (pp. 137-184). Madrid: Traficantes de Sueños.

Alguacil, J. (2008). Espacio público y espacio político: la ciudad como el lugar para las estrategias de participación. Polis: Revista Latinoamericana, 20(10), 199-223.

Arendt, H. (2009). La condición humana. Barcelona: Paidós.

Aymerich, J. (2013). Segregación urbana y políticas públicas con especial referencia a América Latina. Revista de Sociología, 18, 117-130. Recuperado de https://doi.org/10.5354/0716-632X.2004.27803

Benford, R., y Snow, D. (1994). Marcos de acción colectiva y campos de identidad en la construcción social de los movimientos. En J. Gusfield y E. Laraña (Coords.), Los nuevos movimientos sociales: de la ideología a la identidad (pp. 221-252). Madrid: Centro de Investigación Social CIS.

Benhabib, S. (2006). El ser y el otro en la ética contemporánea: feminismo, comunitarismo y posmodernismo. Barcelona: Gedisa.

Berroeta, H. y Vidal, T. (2012). La noción de espacio público y la configuración de la ciudad: fundamentos para los relatos de pérdida, civilidad y disputa. Polis: Revista Latinoamericana, 31, 1-17. 
Borja, J. y Muxi, Z. (2003). El espacio público: ciudad y ciudadanía. Barcelona: Electa. Recuperado de http://www.esdionline.com/repositori/public/dossiers/DIDAC_wdw7ydy1.pdf

Brah, A. (1996). Cartografía de la diáspora: identidades en cuestión. Madrid: Traficantes de Sueños.

Bustelo, M. y Lombardo, E. (Eds.). (2007). Políticas de igualdad en España y en Europa. Madrid: Cátedra.

Cabello, A. M. (2015). Caracterización urbana de Talca: ciudad intermedia de Chile. Nadir: Rev. elect. geogr. Austral, 7(2), 1-22.

Césaire, A. (1994). Cahier d'un retour au pays natal. Ibadan: New Horn Press.

Chihu Amparán, A. (2006). El discurso del EZLN desde la perspectiva del frame analysis. El Cotidiano, 21(137), 62-73. Recuperado de http://www.redalyc.org/articulo.oa?id=32513708

Chihu Amparán, A. (2012). La teoría del framing: un paradigma interdisciplinario. Acta Sociológica, 59(78), 77-101.

Chihu Amparán, A., y Gallegos, A. L. (2004). El análisis de los marcos en la obra de William Gamson. Estudios Sociológicos, 22(2), 435-460.

Delgado, M. (2001). Memoria y lugar: el espacio público como crisis de significado. Tránsitos: espacio público, masas corpóreas. Valencia: General de Ediciones de Arquitectura. Recuperado de https://www.tccuadernos.com/territorio-ciudad/23memoria-y-lugar-el-espacio-publico-manuel-delgado.html

Delgado, M. y Malet, D. (2011). El espacio público como ideología. Urbandoc, 1, 57-65.

Di Méo, G. (1998). Géographie sociale et territoire. París: Nathan Université.

Duffy, J. (s/f). Biblioteca Humana. Recuperado de https://www.rootsandshoots.org/sites/default/files/RSPeaceDayHumanLibraryES.pdf

Fanon, F. (2009). Piel negra, máscaras blancas. Madrid: Akal.

Fraser, N. (1990a). ¿Qué tiene de crítica la teoría crítica? Habermas y la cuestión del género. En S. Benhabib, D. Cornell y Institució Valenciana d'Estudis i Investigació (Eds.), 
Teoría feminista y teoría crítica: ensayos sobre la política de género en las sociedades de capitalismo tardio (pp. 49-88). Valencia: Edicions Alfons el Magnànim: Institució Valenciana d'Estudis i Investigació.

Fraser, N. (1990b). Rethinking the Public Sphere: A contribution to the critique of actually existing democracy. Social Text, 25-26, 56-80. Recuperado de https://doi.org/10.2307/466240

Fraser, N. (1997). Justice Interruptus: Critical reflections on the "postsocialist" condition. New York: Routledge.

Fraser, N. (2006). La justicia social en la era de la política de identidad: redistribución, reconocimiento y participación. Revista de Trabajo, 4(6), 83-99. Recuperado de http://trabajo.gob.ar/downloads/igualdad/08ago-dic_fraser.pdf

Gamson, W. (1985). Goffman's Legacy to Political Sociology. Theory and Society, 14(5), 605-622. Recuperado de https://doi.org/10.1007/BF00160018

Gamson, W. (1992). Talking Politics. Cambridge: Cambridge University Press.

Gaytan Sánchez, P. (2009). Del piropo al desencanto: un estudio sociológico. Ciudad de México: Universidad Autónoma Metropolitana, Unidad Azcapotzalco. Recuperado de http://zaloamati.azc.uam.mx/handle/11191/1855

Hill Collins, P. (2009). Black Feminist Thought: Knowledge, Consciousness, and the Politics of Empowerment. New York: Routledge.

Human Library (2016). Human Library FAQ. Recuperado de http://humanlibrary.org/faqhuman-library/

Instituto Nacional de Estadísticas (2018). Censo 2017. Recuperado de: https://resultados.censo2017.cl/Region?R=R07

Lefebvre, H. (1978). El derecho a la ciudad (4a ed.). Barcelona: Península.

Little, N., Nemutlu, G., Magic, J. y Molnár, B. (2011). Don't Judge a Book by its Cover! The Living Library Organiser's Guide 2011. Paris: Council of Europe Publishing. 
McAdam, D., McCarthy, J. D. y Zald, M. N. (Eds.) (1999). Movimientos sociales: perspectivas comparadas: oportunidades políticas, estructuras de movilización y marcos interpretativos culturales. Madrid: ISTMO.

Micheletti, S. (2016a). Caracterización del flujo inmigratorio en la Región del Maule, Chile. Nuevas tendencias y proyecciones. En S. Micheletti (Ed.), Cuadernos de Sociología UCM Vol. 1 (pp. 43-50). Talca: Escuela de Sociología UCM. Recuperado de https://issuu.com/sociologiaucm/docs/cuadernosescum-v1n2

Micheletti, S. (2016b). Inmigración en la ciudad intermedia agraria: el caso de Talca. Rumbos TS, 14, 11-28.

MIGRA (2014). Plan Estratégico Colectivo MIGRA. Manuscrito no publicado. Colectivo Migra, Chile.

Ministerio del Interior y Seguridad Pública (2016). Migración en Chile 2005-2014. Santiago: Ministerio del Interior y Seguridad Pública.

Okin, S. M. (1989). Justice, Gender and the Family. New York: Basic Books.

Raffestin, C. (1980). Pour une géographie du pouvoir. Paris: Librairies Techniques.

Sabatini, F., Cáceres, G. y Cerda, J. (2001). Segregación residencial en las principales ciudades chilenas: tendencias de las tres últimas décadas y posibles cursos de acción. EURE, 27(82), 21-42. Recuperado de https://doi.org/10.4067/S025071612001008200002

Sabatini, F. y Soler, F. (1995). Paradoja en la planificación urbana en Chile. Revista EURE, 62, 61-73.

Sanhueza, S., Salas, S. y Ramírez, D. (2016). Narrativas de otros ojos. Testimonios de mujeres migrantes en la región del Maule. Talca: Universidad Católica del Maule.

Segovia, O. y Dascal, G. (Eds.). (2000). Espacio público, participación y ciudadanía. Santiago: $\quad$ Ediciones $\quad$ Sur. Recuperado de http://www.sitiosur.cl/publicacionescatalogodetalle.php?PID=2712

Snow, D. A. y Benford, R. D. (2006). Ideología, resonancia de marcos y movilización de participantes. En A. Chihu Amparán (Ed.), El "Análisis de los marcos" en la sociología de los movimientos sociales (pp. 83-118). Ciudad de México: Porrúa, UAM- Iztapalapa. 
Turner, V. W. (1997). The Ritual Process: Structure and Anti-structure. New Brunswick: Aldine Transaction.

Vallejo, E. y Rivarola, M. P. (2013). La violencia invisible: acoso sexual callejero en Lima Metropolitana y Callao. Cuadernos de Investigación IOP, 4, 1-19. Recuperado de http://repositorio.pucp.edu.pe:8085/index/handle/123456789/34946

Van Gennep, A. (1960). The Rites of Passage. Chicago: University of Chicago Press.

Volante, P. (2011). Una antropología relevante: la "condición humana" desde Hannah Arendt. Pensamiento educativo. Revista de Investigación Educacional Latinoamericana, 28(1), 85-104.

Young, I. M. (1990). Imparcialidad y lo cívico público. Algunas implicaciones de las críticas feministas a la teoría moral y política. En S. Benhabib y D. Cornell (Eds.), Teoría feminista y teoría crítica: ensayos sobre la política de género en las sociedades de capitalismo tardío (pp. 89-118). Valencia: Edicions Alfons el Magnànim.

Young, I. M. (1996). Vida política y diferencia de grupo: una crítica del ideal de ciudadanía universal. En C. Castells (Ed.), Perspectivas feministas en teoría política (pp. 99126). Barcelona: Paidós Ibérica.

Young, I. M. (2000). La justicia y la política de la diferencia. Barcelona: Cátedra.

Zald, M. N. (1999). Cultura, ideología y creación de marcos estratégicos. En D. McAdam, J. D. McCarthy y M. N. Zald (Eds.), Movimientos sociales, perspectivas comparadas: oportunidades políticas, estructuras de movilización y marcos interpretativos culturales (pp. 369-388). Madrid: ISTMO.

Cómo citar este artículo: Micheletti, S., Vera, S. y Cubillos, J. (2018). La Biblioteca Humana Migrante: uso del espacio público y resignificación del proceso migratorio. Si Somos Americanos. Revista de Estudios Transfronterizos, 18(2), 53-77. 